\title{
Le réel et le rationnel. Rationalité et conséquences inattendues chez Max Weber
}

Mohamed Cherkaoui

\section{(2) OpenEdition}

\section{Journals}

Édition électronique

URL : http://journals.openedition.org/ress/372

DOI : $10.4000 /$ ress.372

ISSN : $1663-4446$

Éditeur

Librairie Droz

Édition imprimée

Date de publication : 1 mars 2004

Pagination : 73-86

ISBN : 2-600-00941-8

ISSN : 0048-8046

\section{Référence électronique}

Mohamed Cherkaoui, « Le réel et le rationnel. Rationalité et conséquences inattendues chez Max Weber », Revue européenne des sciences sociales [En ligne], XLII-129 | 2004, mis en ligne le 01 mars 2004, consulté le 19 avril 2019. URL : http://journals.openedition.org/ress/372 ; DOI : 10.4000/ ress.372 


\section{Mohamed CHERKAOUI}

\section{LE RÉEL ET LE RATIONNEL. Rationalité et conséquences inattendues chez Max Weber*}

Dans son essai Protestantisme et capitalisme, Philippe Besnard (1970) plaide, avec science et rigueur, la cause de la cohérence logique de la thèse wébérienne que certains critiques avaient mise en doute. Il a, de surcroît, appelé l'attention sur l'importance des changements de la théologie calviniste pour bien comprendre cette cohérence et la portée du dogme de la prédestination dans l'économie générale de la plus célèbre œuvre de Weber. Il a, par ailleurs, noté que l'éthique et l'esprit du capitalisme sont des conséquences non prévues et non voulues par les réformateurs et exigent logiquement une explication.

Est-il possible de déduire du dogme de la prédestination toutes les propositions relatives à l'ascétisme protestant en tant que code moral d'une part, à l'esprit du capitalisme d'autre part, comme conséquences non voulues et non prévues? Plus généralement, ces effets non intentionnels forment-ils des épiphénomènes, des sous produits des actions humaines, ou bien constituent-ils au contraire une classe de phénomènes d'une importance telle que Weber leur accorde une place centrale dans sa théorie générale de l'action et le plus grand soin pour rendre intelligible leur émergence? Leur existence ne conforte-t-elle pas plutôt l'explication holiste - marxienne ou durkheimienne notamment - selon laquelle les hommes sont le produit de structures sociales qui leur préexistent plutôt que les créateurs de celles-ci? Ne contredisent-ils pas le principe d'individualisme méthodologique? Ne remettent-ils pas en cause le principe même de rationalité comme étant pratiquement illusoire? Nous verrons qu'il n'en est rien ${ }^{1}$.

Dans un célèbre passage de L'éthique protestante, Weber affirme que l'on pourrait déduire logiquement du rationalisme protestant tout ce qui caractérise la civilisation moderne. Cette contribution se veut une reconstruction schématique de la démonstration, qui est en filigrane dans cet ouvrage, et surtout une enquête

* Je remercie vivement Raymond Boudon, Massimo Borlandi, Max Engammare et Werner Gephart de leurs précieux commentaires et suggestions.

Il n'est pas inutile de rappeler ici que l'existence des conséquences inattendues et parfois non voulues de l'action a été signalée bien avant Weber et étudiée de façon plus ou moins systématique après lui. Citons notamment la parabole de la fable des abeilles de Mandeville (1705), la métaphore de la main invisible chez Adam Smith (1759) (1776) et chez de nombreux philosophes écossais, la ruse de la raison dans l'histoire chez Hegel (1821) (1837) que l'on peut considérer comme une amplification du mécanisme smithien. Plus tard, on retrouvera l'idée amplement développée chez Meinecke (1936), Merton (1936), Popper (1945) et Boudon (1977). Il n'entre pas dans notre propos de montrer dans ce court texte l'apport inédit de Weber en dehors de quelques indications faites au passage et que nous espérons développer plus tard. 
minutieuse, quoique peut-être inachevée, sur les mécanismes de production des conséquences inattendues des actions ${ }^{2}$.

\section{COHÉRENCE RATIONNELLE DU DOGME RELIGIEUX ET SES CONSÉQUENCES}

Il suffit d'ouvrir L'éthique protestante pour se rendre immédiatement compte que l'usage des mots «rationnel», «rationalité», « rationalisation», est fréquent. Cependant, nulle part dans cet essai, on ne trouvera les expressions de «Wertrationalität», «wertrational», «Zweckrationalität» et «zweckrational». C'est en effet bien plus tard que Weber (1913) forgera ces concepts en particulier dans son Essai sur quelques catégories fondamentales de la sociologie compréhensive. Nous savons pourtant que L'éthique protestante est entièrement construite autour de la notion de rationalité axiologique qui se distingue de la rationalité instrumentale ou en finalité. La rationalité instrumentale se définit en fonction de la relation congruente entre les moyens et les fins, comme des conséquences théoriquement prévisibles que l'acteur prend en compte dans ses calculs (motifs utilitaires) pour entreprendre une action. Pour sa part, la rationalité axiologique renvoie aux valeurs qui, seules, rendent intelligibles l'action sans égard pour les conséquences qui risquent de découler de la décision de l'agent (motifs idéaux, identification personnelle ou engagement vis à vis d'idéaux moraux).

Écartons d'emblée la position ontologique d'un Hegel que Weber considère comme un dogme philosophique qui induit des obstacles à la connaissance scientifique pour autant que le réel n'est pas intrinsèquement rationnel et que sa rationalisation ne peut être disjointe d'une certaine activité humaine. Pour Weber, la rationalisation est double: elle est objective lorsque le chercheur tente de mettre en évidence et d'analyser les motifs de l'acteur et les moyens que ce dernier utilise pour atteindre un objectif qu'il s'assigne; elle est subjective lorsque l'individu explique ou motive ses actes en se réclamant d'une valeur, politique, religieuse, éthique ou autre. Rendre rationnel c'est être capable de produire des procédures, des règles ou des techniques pour introduire une cohérence et une efficacité dans les actions humaines. Or cette double rationalisation est le propre du capitalisme, comme Weber (1920-21) le développe dans l'Avant propos de sa sociologie des religions du monde et comme il le démontre tout au long de L'éthique protestante. En une phrase ramassée bien qu'évidemment incomplète, il résume cette idée dans son Histoire économique p. 302: «ce qui a en fin de compte produit le capitalisme consiste en l'entreprise rationnelle douée de durée, en la comptabilité rationnelle; en la technique rationnelle, dans le droit rationnel, mais non pas exclusivement; il faut ajouter à titre de complément la conviction rationnelle, la rationalisation de la conduite de la vie et l'ethos économique rationnel.»

La déduction à partir du rationalisme protestant de tout ce qui concerne la civilisation moderne est logiquement possible mais, comme le remarque Weber $(1905,248)$, cela supposerait l'unité du «psychisme collectif qui serait réductible à une formule. Mais elle appartient du domaine du possible, intellectuellement s'entend, exactement comme une interprétation strictement matérialiste. 
Dans le même Avant-propos, la rationalité est également entendue comme idée tendancielle qui caractérise l'histoire de l'Occident. Mais ce sont des circonstances historiques qui font qu'elle se réalise. La question posée par Weber est celle de la détermination des traits distinctifs du rationalisme occidental et d'en expliquer l'origine. Or ce rationalisme dépend tout à la fois du rationalisme économique et de celui des dispositions qu'ont les hommes d'adopter certains comportements qui peuvent soit encourager le rationalisme économique soit l'inhiber.

En quoi consiste le rationalisme protestant? On sait que la doctrine de la prédestination a pris dans la pensée de Calvin une importance de plus en plus grande, en partie à la suite des objections nombreuses qu'elle soulevait. Elle est à peine mentionnée dans l'édition de 1536 de L'institution de la religion chrétienne. Elle a par la suite pris progressivement une place croissante dans les éditions suivantes jusqu'à occuper sous le titre «De l'élection éternelle» quatre chapitres du livre III de la dernière édition. Dans son ultime formulation, la doctrine de la prédestination découle donc non d'expériences religieuses mais des nécessités logiques de la pensée de Calvin. Weber $(1905,119)$ note à maintes reprises cette amplification «de la cohérence logique d'une méditation religieuse orientée vers Dieu, non vers les hommes». Et c'est précisément cette "grandiose cohérence» qui a tant fasciné les individus. En d'autres termes, outre le contenu dogmatique qui offre des solutions à des problèmes théologiques majeurs restés en suspens, c'est aussi l'aspect formel et la rigueur logique de la construction calvinienne qui a séduit cette génération. A partir d'un postulat, elle a été en mesure de déduire, more geometrico pour ainsi dire, une série de propositions non contradictoires sur les rapports entre Dieu et les hommes et sur les devoirs de ces derniers. Une fois que l'on admet l'axiome du dogme de la prédestination, les théorèmes qui en découlent semblent s'imposer à l'esprit aussi nécessairement que ceux de la géométrie, comme Boudon (1998) le souligne fortement. Weber signale lui-même à plusieurs endroits la «cohérence logique» de la pensée calvinienne, sa rigueur, dont la rationalité constituera en fait le modèle pour les croyances et les comportements des puritains. Et c'est bien elle aussi qui constitue «ce point final de ce vaste processus de désenchantement du monde » qui a débuté avec le judaïsme et la pensée scientifique.

Résumons à grands traits. Calvin reprend, entre autres, la thématique augustinienne sous sa forme du double décret (Weber 1905, 117 n): Dieu a décidé d'élire certains et de damner les autres librement, de toute éternité, avant même la création et donc avant le pêché originel, selon sa volonté, de façon gratuite ${ }^{3}$. La grâce de Dieu ne peut ni se perdre ni se gagner. Les élus comme les damnés ne peuvent avoir aucune influence sur la décision divine quelles que soient leurs bonnes œuvres. Ce decretum horribile, irrévocable, est celui d'un Dieu transcendant que l'entendement humain est dans l'incapacité de comprendre.

Le texte de Calvin dit: « Nous disons que le Seigneur a une fois constitué en son conseil éternel et immuable, lesquels il voulait prendre à salut, et lesquels il voulait laisser en ruine. Ceux qu'il appelle à salut, nous disons qu'il les reçoit de sa miséricorde gratuite (souligné par MC), sans avoir égard aucun à leur propre dignité. Au contraire que l'entrée de vie est forclose à tous ceux qu'il veut livrer en damnation, et que cela se fait par son jugement occulte et incompréhensible 
A partir de ce postulat découle logiquement un ensemble de conséquences dont nous ne retenons ici que les plus importantes ${ }^{4}$.

La prédestination engendre chez le calviniste une solitude intérieure. Il est seul sinon à tenter de décoder le décret de Dieu relatif à son salut éternel du moins à suivre son destin. Rien ne peut lui venir en aide: ni prédicateur, ni sacrement, ni institution, ni Eglise, ni même Jésus, qui n'est mort que pour les élus (Weber 1905, 120 et sq.), ne sont des moyens d'obtenir la grâce. La confession individuelle comme moyen de soulager sa conscience lui étant refusée, il est condamné à conduire sa vie quotidienne comme une lutte sans trêve pour déceler les signes de sa grâce ${ }^{5}$. Dans la pratique toutefois, il existe plusieurs critères qui lui permettent de reconnaître qu'il est élu. Le premier est la foi dans son élection: le doute, expression de l'insuffisance de la foi, n'est pas permis à des saints. Le second est une confiance en soi à laquelle on accède par le travail sans relâche qui donne sinon la certitude du moins une sorte d'indice incertain de la grâce, travail qui est aussi psychologiquement un moyen pour réagir contre l'angoisse religieuse du calviniste provoquée par l'impossibilité de connaître le décret de Dieu le concernant. Le troisième est un type de conduite, l'exigence d'une qualité de l'action et le contrôle méthodique de sa vie qui permettent au chrétien d'augmenter la gloire de Dieu. Les bonnes œuvres ne sont pas des moyens pour obtenir le salut mais uniquement des signes d'élection, des moyens techniques pour se délivrer de l'angoisse du salut.

Les relations sociales et le caractère rationnel de la morale puritaine découlent du dogme et de ses conséquences. En premier lieu, c'est la doctrine de la prédestination qui libère les énergies de l'individu pour transformer le monde qui n'existe que pour servir la gloire de Dieu (Weber 1905, 127 n. 31). En second lieu, toute activité de l'homme ici bas ne doit avoir qu'un seul but, celui d'augmenter cette gloire. On exige des hommes et de toutes les institutions humaines qu'ils soient efficaces socialement: d'où la supériorité du puritain en matière d'organisation sociale. L'un des corollaires de cette proposition est que la recherche du bien public est préférable à celle de tout bien personnel, parce qu'elle est socialement plus efficace. L'amour du prochain ne doit cependant

Il convient de rappeler ici que la doctrine de la prédestination n'a pas été acceptée par tous les mouvements ascétiques. Si le piétisme peut être considéré comme la continuation de la doctrine de Calvin et une reviviscence du principe de la prédestination sous une forme adoucie, il soulignait aussi le côté sentimental de la religion qui était étranger au calvinisme. Mis à part une forme extrémiste, le piétisme conservait les principes ascétiques et le renforcement de l'éthique professionnelle dont nous parlerons plus bas au sujet de la doctrine calviniste pure. Le méthodisme, pour sa part, qui visait principalement les masses, se caractérisait par ce sentimentalisme et une indifférence aux fondements dogmatiques mais gardait la nature méthodique de la conduite rationnelle qu'il avait héritée de l'ascétisme puritain. Il trouvait dans l'aspiration à une régénération - certitude du salut comme résultat de la foi et fondement de la grâce - le substitut du principe de la prédestination. Cette poursuite d'une vie plus élevée se traduisait également par la recherche rationnelle de la perfection dans ce monde.

Il s'agit, soulignons le, de la confession individuelle qui n'est pas un sacrement et non de la confession collective des péchés qui était pratiquée. Par ailleurs, si la recherche de la certitude du salut est en partie étrangère à Calvin, elle est en revanche fondamentale chez les calvinistes et presbytériens, comme l'indique Weber qui, rappelons le, prend appui sur les épigones pour asseoir sa thèse plutôt que sur le texte même de Calvin. 
s'inscrire qu'au service de Dieu et toute idolâtrie de l'autre est prohibée. Une autre conséquence de ce dogme est son caractère antiautoritaire: toute intervention de l'église ou de l'État en matière éthique ou de salut est interdite (Weber 1905, 125 n. 29). Historiquement, on note bien une relative grande immunité des peuples puritains à l'égard du césarisme, une égalité réelle entre les hommes dans sa forme la plus extrême, et un penchant pour la démocratie que nous examinerons plus bas.

Le second corollaire important est relatif à l'activité professionnelle qui participe du même principe, entendons l'activité professionnelle au service de l'utilité sociale impersonnelle. Le puritain doit se borner à un travail spécialisé, renoncer dès lors à la prétention à l'universalisme faustien. La division du travail est nécessaire dans la mesure où elle accroît l'habileté et donc la production. Le chrétien doit exister pour son entreprise non l'inverse. Le patron comme l'ouvrier traiteront le travail comme vocation: c'est même le but de la vie.

L'ascétisme protestant a eu pour effet de débarrasser le puritain des inhibitions de l'éthique traditionaliste qui freine le désir d'acquérir. Bien plus, l'acquisition devient un impératif catégorique. L'homme devient une machine à acquérir, non à consommer. L'accumulation rationnelle et légale des richesses par le travail et l'épargne est légalisée puisqu'elle est voulue par Dieu. Elle exclut par conséquent l'enrichissement malhonnête et toute consommation ostentatoire ou même superflue. Les vertus cardinales sont l'honnêteté, la ponctualité, l'application au travail, la frugalité, un usage rationnel et économe du temps.

Pour avoir traité la question ailleurs (Cherkaoui 2003), nous n'insisterons pas sur la définition du rationalisme du comportement économique qui découle indirectement de la rationalité du dogme et directement de celle de l'éthique via le mécanisme de l'épreuve. Rappelons que Weber établit que les mêmes contenus se trouvent dans l'esprit du capitalisme moderne et dans l'ascétisme protestant. On retrouve, ici et là, les mêmes éléments essentiels mais qui sont dépourvus du fondement religieux. L'esprit du capitalisme est une conduite rationnelle fondée sur l'idée de Beruf, qui est née de l'ascétisme protestant. L'équivalence de «l'esprit du capitalisme» et de «la culture capitaliste» a été soulignée notamment par Gephart (1998, 175-181). Gagner plus d'argent grâce à son travail et à sa compétence professionnelle, ce qui est le contraire de l'homme d'affaire sans scrupules, accumuler sans consommation excessive mais en réinvestissant, gérer son entreprise selon un nouvel esprit qui refuse la tradition, utilise les règles de la comptabilité, contrôle toutes les étapes de la production et de la commercialisation, améliore constamment la qualité et le volume de la production, réduit les coûts, bref recherche le profit maximum. Par ailleurs, l'uniformisation de la vie du puritain prépare la standardisation de la production capitaliste. «La conception puritaine de l'existence a veillé sur le berceau de l'homo oeconomicus moderne», conclut Weber (1905, p. 236).

Nous avions montré ailleurs les mécanismes qui rendent possible le passage du dogme et de l'éthique à l'action (Cherkaoui, 2003). Toutefois, nous avions tout juste signalé, chemin faisant, l'existence du problème fondamental des conséquences inattendues du dogme et de son explication sur lesquels il convient à présent de se pencher. Une chose est sûre: rien n'est assurément plus éloigné des intentions premières des réformateurs et des projets qu'ils visaient d'atteindre que l'élaboration de règles éthiques et la codification des comportements écono- 
miques et sociaux ${ }^{6}$. Le dogme religieux ne se préoccupe que du salut de l'âme et de lui seul. L'éthique ascétique et l'esprit du capitalisme sont par conséquent des conséquences inattendues (Weber 1905, 106). Pourquoi de tels phénomènes apparaissent-ils? C'est, nous semble-t-il, l'un des problèmes centraux de la théorie générale de l'action que Weber soulève pour la première fois dans L'éthique protestante.

\section{TYPOLOGIE DES SOURCES D'ÉMERGENCE DES CONSÉQUENCES NON PRÉVUES}

Il serait sans doute pédagogiquement utile de construire une typologie, fût-elle provisoire, des conditions d'émergence des conséquences inattendues et parfois non voulues par les acteurs, que Weber (1922 b, 353) qualifie de «Parodoxie der Folgen », de paradoxe des conséquences ${ }^{7}$. A cette fin, il sera nécessaire de solliciter, outre L'éthique protestante, les autres œuvres de Max Weber où de tels phénomènes sont analysés. S'il est vrai que ces effets concernent tous les types d'action, ils constituent cependant davantage une énigme pour les actions rationnelles en finalité que pour celles qui relèvent de la rationalité axiologique. Puisque, en effet, les actions axiologiquement rationnelles ne se rapportent qu'à des valeurs ultimes et qu'elles sont exécutées sans égard pour les conséquences qu'elles engendrent pour l'acteur et pour autrui, leur caractère parfois inattendu ne représente, en fait, une énigme que pour le sociologue et non pour l'acteur. Peu importe que l'action se retourne contre celui qui a pris la décision d'agir et le détruit du moment qu'il accepte de se conformer à l'éthique du Sermont sur la montagne qu'il tient pour une valeur qui donne sens à sa vie.

Cette affirmation est inexacte historiquement si on la prend à la lettre et qu'on l'applique à Calvin. Il est absolument incontestable que ce dernier a été à l'origine d'un ordre moral qu'il appliquait avec la plus sévère fermeté. Calvin a, comme l'on sait, créé le Consistoire pour exercer un contrôle social total sur tous les comportements de la population genevoise. Le Consistoire a fonctionné comme une cour de justice et comme une institution éducative. Certains historiens, Monter (1976) par exemple, pensent que près du 1/15 des adultes de la cité lémanique est passé devant le Consistoire. Sur le même thème, on se référera au chapitre premier de Kindon (1995). Une fois cette précision faite, il convient de ne pas oublier que Max Weber $(1904,106)$ ne vise pas ce qu'il appelle «les créations de Calvin», mais les réformateurs à propos desquels il écrit: "Nous ne croyons certes pas que la recherche des biens de ce monde, conçue comme une fin en elle-même, ait jamais revêtu une valeur éthique pour aucun d'entre eux. Disons-le une fois pour toutes: pour aucun des réformateurs - et parmi eux nous rangerons un Mennon, un Fox, un Wesley - les programmes de réforme morale n'ont jamais constitué la préoccupation dominante... Leurs buts éthiques, les manifestations pratiques de leurs doctrines étaient tous ancrés là (le salut des âmes), et n'étaient que les conséquences de motifs purement religieux. C'est pourquoi nous devons nous attendre à ce que les effets de la Réforme sur la culture (...) aient été des conséquences imprévues, non voulues, de l'œuvre des réformateurs, conséquences souvent fort éloignées de tout ce qu'ils s'étaient proposé d'atteindre, parfois même en contradiction avec cette fin ». (les mots soulignés le sont par Weber lui-même).

C'est à propos de la relation entre le caractère rationnel de l'ascèse et l'accumulation des biens que Weber (1922 b) emploie l'expression de paradoxe : «In eigentümlicher Paradoxie gerät vor allen Dingen, wie schon mehrfach erwänt, die Askese immer wieder in den Widerstreit, dass ihr rationaler Charakter zur Vermögensakkumulation führt». La même expression se trouve à d'autres endroits dans Weber (1922 b). 
Or il en va tout autrement des actions qui relèvent de la rationalité instrumentale. Elles supposent d'une part que la congruence des moyens et des fins soit, en principe, bien définie; elles postulent d'autre part que les conséquences des actions soient prises en compte dans le calcul de l'acteur. Pourquoi dès lors de telles actions engendrent-elles souvent des effets inattendus? Les conditions dans lesquelles ces derniers apparaissent sont au moins au nombre de quatre. La première [1] apparaît lorsque, en dépit de l'adéquation des moyens et des fins, l'action déborde le domaine d'activité dans lequel elle est sensée s'appliquer. La seconde [2] dérive de l'apparition d'un conflit entre les rationalités de sphères relativement autonomes. La troisième [3] découle de l'irruption de nouvelles valeurs durant le processus de réalisation qui entrent en conflit avec celles sur lesquelles repose la décision. La quatrième [4] enfin résulte d'une inadéquation entre les moyens utilisés et les fins poursuivies: si donc il y a ignorance ou erreur, les prévisions risquent d'être fausses et les conséquences de l'action inattendues, et elles sont d'autant plus éloignées des objectifs de l'agent que peuvent apparaître des processus cumulatifs entre cette inadéquation et les effets de l'extension, du conflit de rationalités ou de l'irruption de nouvelles valeurs ${ }^{8}$. Bien entendu, ces quatre types purs sont susceptibles de se combiner entre eux et de produire des figures mixtes d'une complexité redoutable pour l'analyse, en particulier les types $[4+1],[4+2],[4+3]$.

Le tableau suivant résume les cas où apparaissent de tels phénomènes.

\begin{tabular}{|l|c|c|c|c|}
\hline \multirow{4}{*}{} & stricte & $\begin{array}{c}\text { extension } \\
\text { du domaine }\end{array}$ & $\begin{array}{c}\text { conflit de } \\
\text { rationalités }\end{array}$ & $\begin{array}{c}\text { irruption de } \\
\text { nouvelles valeurs }\end{array}$ \\
\hline \multirow{4}{*}{ Relations } & \multicolumn{4}{|c|}{ Adéquation } \\
\cline { 2 - 6 } & $\begin{array}{c}\text { science/ } \\
\text { technique }\end{array}$ & CINV & CINV & CINV \\
\hline \multirow{5}{*}{ Inadéquation } \\
Moyens/fins
\end{tabular}

Légende $: C I N V=$ conséquences inattendues et parfois nous voulues.

Il est inutile d'alourdire l'exposé en étudiant en détail la quatrième source dont l'évidence n'échappe à personne. Puisque, en effet, l'acteur ne dispose jamais

s Cette typologie a incontestablement fortement influencé celle que Merton (1936) propose dans sa célèbre étude. Merton distingue cinq raisons de l'apparition des conséquences inattendues. La première est l'ignorance puisque aucun acteur ne dispose d'une information parfaite. La seconde est l'erreur qui peut concerner aussi bien l'évaluation de la situation que l'usage que nous en faisons pour définir l'objectif ou la sélection du type d'action à exécuter et enfin l'exécution de l'action elle-même. La troisième est l'urgence de l'intérêt immédiat qui recoupe en partie l'extension du domaine selon Weber. La quatrième renvoie aux valeurs au sens wébérien que Merton n'analyse pas de façon aussi élaborée que Weber. La cinquième et dernière est la prédiction qui s'autodétruit. La prédiction marxienne de la concentration progressive des richesses et la paupérisation des masses par exemple a influencé les actions menées par les syndicats pour réduire cette paupérisation sinon pour l'éliminer. La conséquence est qu'elle n'a pas eu lieu contrairement à la prédiction. 
d'une information complète et parfaite sur la situation, les moyens, les objectifs et les conséquences de son action, il n'y a guère lieu de s'étonner de voir surgir des effets inattendus. Soulignons toutefois que l'action demeure rationnelle même si l'information est imparfaite. Examinons les autres sources.

1/ Analysons quelques exemples wébériens où l'action même rationnelle dans une sphère dépasse les frontières de ce domaine et engendre des effets inattendus et parfois non voulus. Nous traiterons plus particulièrement certaines conséquences de la neutralité de la science, celles de la rationalité de la théodicée calviniste, enfin les effets du puritanisme sur la démocratie. L'exemple des effets de la bureaucratisation rampante de nombreux domaines est trop connu pour que l'on ne s'y arrête pas.

Considérons le problème de la neutralité de la science et de ses effets. La science constitue un des éléments les plus importants de la rationalisation. Elle nous apprend que, théoriquement du moins, il nous est possible de maîtriser la nature ou d'exercer une influence sur la société grâce au calcul et à la prévision fondés sur la connaissance objective des phénomènes. Depuis le XVII siècle, ses réussites constantes ont valu à sa démarche et à sa méthode d'être prises pour modèle. Elle a exercé une influence non seulement sur les sociétés mais aussi sur les croyances des hommes. En offrant un modèle aux activités rationnelles par finalité, elle a contribué à la rationalisation des activités non scientifiques. Mais, du même coup, elle a chassé du monde toutes les puissances mystérieuses et banni tout usage des moyens magiques, participant ainsi, avec d'autres, à ce désenchantement du monde qui n'était pas contenu dans ses objectifs (Weber 1905, 122; Weber 1919, 77-79). Ce résultat final de l'activité du savant dépasse ses intentions.

D'autres conséquences inattendues en découlent. Il en est ainsi de «l'anomie» qui caractérise l'homme moderne confronté à l'infinitude du progrès, à la dure réalité du provisoire et à l'impossibilité de combler ses désirs intellectuels et sensuels. «L'homme civilisé, écrit Weber (1919, 79-80), au contraire, placé dans le mouvement d'une civilisation qui s'enrichit continuellement de pensées, de savoirs et de problèmes, peut se sentir «las» de la vie et non pas «comblé» par elle. En effet il ne peut jamais saisir qu'une infime partie de tout ce que la vie de l'esprit produit sans cesse de nouveau, il ne peut saisir que du provisoire et jamais du définitif. C'est pourquoi la mort est à ses yeux un événement qui n'a pas de sens. Et parce que la mort n'a pas de sens, la vie du civilisé comme telle n'en a pas non plus, puisque du fait de sa «progressivité» dénuée de signification elle fait également de la vie un événement sans signification.»

A l'évidence, ce texte rejoint certains passages du Suicide où Durkheim propose une des définitions possibles de l'anomie. Incertain de son avenir, incapable d'embrasser le cercle du savoir en le totalisant, impuissant à atteindre les frontières de plus en plus reculées du marché économique ou de toute sphère des activités humaines, inassouvi et insatisfait comme le sont Faust, Don Juan et l'entrepreneur capitaliste, l'homme moderne est «cet abîme sans fond que rien ne peut combler», selon l'expression de Durkheim. Mais cette incertitude que produit le progrès même de la connaissance est fatale à l'individu comme elle l'est à Hamlet qui sombre dans le scepticisme et devient malade «sous l'ombre pâle de la pensée», comme le dit poétiquement Hume (1752). 
Le deuxième exemple des conséquences inattendues et non voulues par extension du domaine d'application est le cas de la doctrine de la prédestination et ses effets sur la morale. C'est là le cœur de la démonstration wébérienne de L'éthique protestante. L'ethos puritain comme l'esprit du capitalisme moderne ne sont en aucune manière contenus dans le programme théologique de Calvin et des réformateurs en général. Pourtant, comme nous l'avons vu dans la première partie de cette étude, leur rationalisme et leur existence même ne se laissent comprendre que comme conséquences inattendues et non voulues du dogme calviniste. Il y a lieu ici de mettre en évidence un double paradoxe qui traduit la combinaison de la première source, entendons une extension de la rationalité religieuse vers des domaines profanes, et l'irréductibilité de valeurs conflictuelles. Le premier paradoxe tient au fait que le dogme calviniste a engendré des effets inattendus puisqu'il est, comme l'écrit Weber $(1905,237)$ paraphrasant Goethe, «cette force qui toujours veut le bien et toujours crée le mal, ce mal qui, pour lui, était représenté par la richesse et ses tentations ». Le second paradoxe exprime l'échec du calvinisme, en tant que théodicée, de rationaliser totalement l'irrationnel de la vie dont l'une des expressions est précisément l'existence de ces effets inattendus.

L'un des objectifs, peut être le plus central, de toutes les théodicées est de rationaliser le monde tel qu'il est perçu et vécu par les hommes qui font l'expérience de son irrationalité, principalement en résolvant le paradoxe des conséquences non voulues de leurs actions. Or, note Weber, les vains efforts des théodicées sont sans doute une preuve éclatante de l'impossibilité de tout rationaliser et de tout prévoir. La théodicée est la réflexion sur la survivance du mal au regard d'un Dieu infiniment bon, omniscient et omnipotent. Comment se fait-il que le mal coexiste avec le bien? Pourquoi Dieu tout puissant et infiniment bon ait pu créer un monde où parfois la souffrance est imméritée et où l'injustice règne? Comment peut-on expliquer et comprendre ce monde dont nous faisons quotidiennement l'expérience de l'incohérence et de l'irrationalité? Si toutes les religions tentent d'y répondre, Weber $(1919,191)$ (1920-1, 572-3) (1922 b, 317-9) semble attacher plus d'importance aux trois théodicées hindoue du karma, du dualisme zarathoustrien, enfin de la prédestination calviniste, parce qu'il pense qu'elles ont déployé les efforts les plus extrêmes pour rationaliser le problème et le résoudre. Le monothéisme, bien qu'il soit un grand essai de rationalisation, n'a pas été en mesure rendre compte d'un paradoxe des conséquences selon lequel les bonnes intentions peuvent avoir des conséquences néfastes. Le monothéisme a échoué devant le pluralisme des valeurs; et c'est ce pluralisme qui rend seul compte de la divergence des lois éthiques, comme l'expérience humaine nous l'apprend.

Le troisième exemple de l'émergence des effets inattendus par extension du domaine concerne la relation entre le puritanisme et la démocratie. Weber lui consacre des aperçus parmi les plus originaux que l'on puisse lire sur ce sujet. Pourquoi les sociétés influencées par l'ascétisme protestant ont-elle été historiquement le berceau de la démocratie moderne? Pourquoi ont-elles été en général hostiles à tout césarisme, à toute autorité? Pourquoi «le jeune Américain, note Weber $(1919,96)$ ne respecte rien ni personne, ni tradition ni situation professionnelle (...) et cela, il l'appelle «démocratie»? Question qu'il avait déjà posée dans son essai sur Les sectes (1906, 263).

On peut déduire du dogme un certain nombre de propriétés relatives aux rapports sociaux qui sont caractéristiques de la démocratie moderne. Bien qu'il ne 
rentre pas dans le projet de Weber de traiter des conséquences politiques du dogme religieux, il ne se prive pas de fournir de nombreuses indications sur ce thème, dont il n'ignorait pas l'importance et le caractère décisif pour l'évolution des sociétés modernes.

Puisque le monde n'existe que pour servir la gloire de Dieu et que seul Dieu règne dans ce monde, toute idolâtrie de la créature, tenue pour attentatoire à la vénération de Dieu, est prohibée (Weber 1905, 127, note 31). Tout comportement et tout sentiment d'allégeance à un homme sont exclus. L'immunité des sociétés puritaines à l'égard du césarisme et des hommes providentiels (ibidem, 127, note 31) a été bien soulignée par le sociologue allemand. Chez les Quakers, le principe est poussé jusqu'à sa limite en refusant les manifestations même de politesse comme le fait de se découvrir, de s'incliner ou d'user du pluriel lorsque l'on s'adresse à une personne de statut social élevé. «On sait, écrit Weber $(1905,190)$, comment ce principe [du refus absolu de l'idolâtrie de la créature] du protestantisme ascétique fonde historiquement le caractère propre de la démocratie contemporaine chez les peuples influencés par le puritanisme en contraste avec celle des peuples d' " esprit latin ». Il constitue aussi, en partie, l'arrière-plan historique des attitudes «irrespectueuses » des Américains, si irritantes ou si réconfortantes selon les uns ou les autres.» Il aurait pu ajouter que l'organisation de L'église calviniste est démocratique puisque ce sont les échelons inférieurs qui élisent en principe leurs délégués à l'échelon supérieur et non comme dans l'église catholique où la hiérarchie est descendante puisqu'elle part du pape pour arriver au curé de paroisse. Il ne serait ni faux ni historiquement dénué d'intérêt de reconnaître dans la hiérarchie ascendante de l'église réformée, principalement chez les presbytériens américains que Weber connaissait pour les avoir observés, un modèle de la structure de la démocratie moderne'.

2/ Tournons-nous à présent vers le deuxième cas des effets inattendus de l'action rationnelle en finalité qui émergent lorsqu'il y a incompatibilité rationnelle des logiques propres à chaque activité et, en définitive, de l'antagonisme des valeurs qui les sous-tendent (Weber 1919, 93). La rationalisation d'une activité, en vertu de sa logique propre, sa «Eigengesetzlichkeit», donc de sa relative autonomie, peut entrer en conflit avec celle d'une autre activité également autonome.

\footnotetext{
Dans une étude savante Mercier (1934) analyse très bien l'apport indirect de la doctrine religieuse de Calvin à la démocratie. Mercier montre notamment comment le principe de l'interprétation individuelle de l'Ecriture et la doctrine de la justification par la foi doivent être tenus pour une étape importante vers l'exaltation de l'individu en tant qu'être rationnel doté d'une âme illuminée par Dieu et donc ouvre la voie à la libre pensée. Si l'on ajoute à cela le fait que pour Calvin l'Eglise doit être une association de fidèles qui élisent leurs représentants et que ces élus ont de fait un pacte d'alliance avec Dieu, on peut incliner à penser que de telles idées ont vraisemblablement contribué indirectement à la formation de la démocratie. Cependant, comme le souligne Mercier $(1934,30)$, «on commettrait une grave erreur en prétendant découvrir en Calvin un précurseur de la démocratie moderne», car il existe chez lui des tendances aristocratiques, élitistes, dirionsnous. Si en effet Calvin est partisan de l'élection des pasteurs par les fidèles, il n'a guère de sympathie pour le suffrage universel. On ne peut dériver de sa pensée politique une théorie de la souveraineté populaire. Il n'y a certes pas chez lui un contrat entre des individus libres qui créent la société ou une institution comme 1'Eglise, pour autant que celle-ci est antérieure à leurs volontés et lui sont par conséquent subordonnés.
} 
Ce qui peut passer pour rationnel pour l'une peut être tenu pour irrationnel pour l'autre. Si donc la rationalisation n'est qu'un point de vue, des conflits peuvent apparaître entre des domaines tout aussi rationnels les uns que les autres. Et, comme le texte de la Zwischenbetrachtung le laisse entendre, la rationalisation croissante des sphères s'accompagne de l'émergence de contradictions de plus en plus radicales entre elles. Il en est de la religion comme de l'économie: chacune a une force logique contraignante qui lui est propre. «Une chose, précise Weber (1905, 53 note 8), n'est jamais «irrationnelle» en soi mais seulement d'un point de vue « rationnel» donné. Pour l'homme irréligieux, vivre de façon religieuse est irrationnel; pour l'hédoniste, l'ascétisme est irrationnel. Peu importe que, mesurés à leurs ultimes valeurs, religion et ascétisme soient une «rationalisation ». Ne serait-il utile qu'à cela, puisse du moins cet essai contribuer à faire comprendre que la simplicité apparente du concept de « rationalité » cache, en fait, sa complexité». Des exemples concrets de cette situation sont légion. Pour n'en prendre qu'un, rappelons-nous les effets calamiteux des possibles contradictions entre les objectifs d'une forme de rationalité propre au système d'enseignement et ceux du marché du travail.

En fait, écrit Weber (1922 a, 397), la rationalisation croissante des domaines les plus variés de l'économie, de la politique, du religieux, ou du militaire par exemple, aboutit à l'effet opposé: «Le progrès que l'on constate dans la différenciation et la rationalisation sociales signifie donc, sinon toujours, du moins normalement quand on considère le résultat, que, dans l'ensemble, les individus s'éloignent de façon croissante de la base rationnelle des techniques et des règlements rationnels qui les concernent pratiquement et, dans l'ensemble, cette base leur est d'ordinaire plus cachée que le sens des procédés magiques du sorcier ne l'est au «sauvage». La rationalisation de l'activité communautaire n'a donc nullement pour conséquence une universalisation de la connaissance relativement aux conditions et aux relations de cette activité, mais le plus souvent elle aboutit à l'effet opposé.»

3/ La troisième source de l'émergence des effets inattendus de l'action est l'irruption des nouvelles valeurs au cours du processus de réalisation de la décision dans les domaines où la rationalité instrumentale prévaut. Cette intrusion traduit une propriété fondamentale de tous les comportements humains et d'une manière générale de tous les systèmes sociaux, entendons leur caractère intrinsèquement ouvert. Nous sommes en mesure de prédire correctement les états futurs de systèmes physiques pour autant que nous disposons des lois et théories qui décrivent et expliquent les relations logiques entre les phénomènes, pourvu aussi que le système soit réellement clos ou que l'hypothèse de sa clôture soit raisonnable, c'est-à-dire qu'il soit fermé à toute influence ou interférence externe qui risque de perturber son fonctionnement ${ }^{10}$. Or cette condition de clôture est rarement satisfaite pour les comportements individuels et les systèmes sociaux. Signalons au

${ }^{10}$ Pour fixer les idées, considérons l'exemple simple des prévisions de la position des planètes du système solaire. Elles se fondent sur les lois et la théorie du mouvement et de la gravitation mais aussi sur des données empiriques antérieures. Ces prévisions ne seront exactes que si le système en question n'est pas perturbé par des interférences externes. Puisque cette hypothèse n'est pas déductible des lois et de la théorie, sa prise en considération est logiquement nécessaire. 
passage que l'interdépendance entre les objectifs poursuivis par l'acteur et ceux, parfois antinomiques, d'un autre agent est un cas particulier de cette intrusion. Et c'est bien le système d'interdépendance entre les sociétaires qui rend compte de nombreuses externalités positives ou négatives. Pour l'essentiel cependant, cette troisième source est réductible à celle, plus générale, du conflit des valeurs. C'est sans doute le mécanisme qui préoccupe le plus Weber et auquel il a consacré les développements les plus importants. Non seulement l'antagonisme des valeurs sévit entre toutes les activités mais il domine également chacune d'elles. Le pluralisme des valeurs est une donnée de l'expérience qu'il est impossible de réduire. Elles sont souvent rivales. Qui ne constate l'antagonisme entre les valeurs de la religion ou de la morale et celles de la politique ou de l'économie? Comment cet antagonisme conduit-il à des effets inattendus? Prenons par exemple l'activité politique. Weber $(1919,180)$ fait remarquer qu' «il est une chose incontestable, et c'est même un fait fondamental de l'histoire, mais auquel nous ne rendons pas justice aujourd'hui: le résultat final de l'activité politique répond rarement à l'intention primitive de l'acteur. On peut même affirmer qu'en règle générale il n'y répond jamais et que très souvent le rapport entre le résultat final et l'intention originale est tout simplement paradoxal ${ }^{11}$. Malgré toutes les rationalisations, l'homme n'est pas maître du devenir. S'il fait l'histoire, il ne maîtrise pas toutes les conséquences de son action.

Or cela s'explique par les conflits entre les valeurs des protagonistes de l'histoire, celles par exemple du chef politique et celles de ses partisans - ou de la bureaucratie - dont il a besoin et sans lesquels nulle action n'est possible. A supposer que la sincérité des convictions du politique soit incontestable, qu'il soit animé de cette passion et de ce dévouement à une cause, s'il veut réaliser ses valeurs de justice ou d'équité, il aura besoin de partisans dont l'engagement à ses côtés s'explique parfois par des raisons strictement matérielles ou des récompenses psychologiques qui sont par conséquent antinomiques aux valeurs pour lesquelles il engage une lutte politique.

Rien d'étonnant à ce que la révolution aux débuts enthousiastes dégénère en général et finisse soit par le despotisme soit par l'émergence d'un nouvel ordre social au bénéfice des partisans ou d'autres qui deviennent des prébendiers. Le chef est donc obligé d'utiliser des moyens qu'en principe il condamne pour autant qu'ils jurent avec la cause qu'il veut défendre. C'est ce qui laisse dire à Weber $(1919,191)$ que «les premiers chrétiens savaient très bien également que le monde était régi par des démons et que l'individu qui se compromettait avec la politique, c'est-à-dire avec les moyens de la puissance et de la violence, concluait un pacte avec des puissances diaboliques; il n'est pas vrai, savaient-ils aussi, qu'au cours de leurs actions le bien engendre uniquement le bien et le mal uniquement le mal: l'on constate bien plutôt et très souvent le phénomène inverse.»

Cette citation fait écho à la fameuse phrase de l'introduction aux Leçons sur la philosophie de l' histoire de Hegel qui écrit: «Il résulte des actions des hommes en général encore autre chose que ce qu'ils projettent et atteignent, que ce qu'ils savent et veulent immédiatement; ils réalisent leurs intérêts, mais il se produit avec cela quelque autre chose qui est caché à l'intérieur, dont leur conscience ne se rendait pas compte et qui n'était pas dans leurs vues (...) La substance de l'action et par suite l'action elle-même se retourne contre celui qui l'a accomplie, elle devient pour lui un choc en retour qui le ruine». Voir aussi Les principes de la philosophie du droit, $2^{\circ}$ partie, $\S \S 118$ et 119. 
L'antagonisme des valeurs ne sévit pas uniquement entre des activités différentes comme la politique, l'économie ou la morale. Il affecte aussi de l'intérieur une même activité. Il y a par exemple au moins deux façons différentes de comprendre la justice. Selon la conception égalitariste de la justice, il convient de donner à chacun selon ses besoins quelles que soient les contributions des sociétaires à la vie collective. Pour une conception méritocratique, la justice distributive doit prévaloir et les rétributions devraient être proportionnelles aux contributions. Les deux conceptions sont moralement aussi légitimes l'une que l'autre, et aucun argument scientifique ne nous autorise à préférer l'une à l'autre. Seule la politique impose un choix qui peut être heureux ou funeste et en contradiction avec la valeur même que l'on prétend servir. Du bien ne sort pas toujours le bien ni le mal toujours le mal.

Ce polythéisme des valeurs ne débouche-t-il pas sur le relativisme? La réponse de Weber (1922a, 428) est dénuée d'ambiguïté: «Le malentendu le plus grossier dont on accable sans cesse ici ou là les intentions des partisans de l'antagonisme des valeurs consiste à interpréter leur point de vue comme un relativisme». Face à l'inconciliabilité des valeurs et aux effets inattendus des actions conduites en fonction d'elles, Weber propose le compromis comme solution dans la mesure où il est le minimum indispensable si l'on veut vivre en commun. Il faut se décider «selon le principe du moindre mal ou celui du relativement meilleur», écrit-il (Weber 1919,99). Ce principe praxéologique traduit un des enseignements de la sagesse des nations et renvoie formellement à l'axiome pessimiste selon lequel il convient toujours de prévoir les pires conséquences de son action. Il retrouve la règle du Maximin de la théorie des jeux. Mais l'énigme des conséquences inattendues est également un défi lancé aux positivistes qui croient en la capacité de la science à prédire tous les effets des actions humaines. Sans doute, fait remarquer Popper $(1945,94)$ avec prudence, nos prévisions s'améliorerontelles à mesure que notre savoir scientifique progressera; mais de nombreuses conséquences imprévisibles de nos actions subsisteront toujours.

GEMAS, CNRS - Université de Paris IV-Sorbonne

mcherkaoui@yahoo.fr

\section{RÉFÉRENCES BIBLIOGRAPHIQUES}

Besnard Ph. (1970), Protestantisme et capitalisme. La controverse post-wébérienne, Paris, Armand Colin.

Boudon R. (1977), Effets pervers et ordre social, Paris, Presses universitaires de France.

- (1998), «La rationalité axiologique», in S. Mesure (ed.),

La rationalité des valeurs, Paris, Presses universitaires de France.

Cherkaoui M. (2003), «Les transitions micro-macro. Limites de la théorie du choix rationnel dans les Foundations of social theory», Revue Française de sociologie, 44 (2), 231-254.

Gephart W. (1998), Handeln und Kultur. Vielfalt und Einheit der Kulturwissenschaften Max Webers, Frankfurt-am-Main.

Hegel G. W. F. (1821), Grundlinien der Philosophie des Rechtes. Trad. fr., Principes de la philosophie du droit, Paris, Vrin, 1975. 
- (1837), Vorlesungen über die Philosophie der Geschichte. Trad. fr., Leçons sur la philosophie de l' histoire, Paris, Vrin, 1967

Hume D. (1752), Inquiry Concerning the Principles of Morals, Indianapolis, Hackett Pub., 1987.

Kindon R. M. (1995), Adultery and Divorce in Calvin's Geneva, Cambridge, Harvard University Press.

Mandeville (1705), The Fable of the Bees or Private Vices, Public Benefits. Trad. fr., La fable des abeilles ou les vices privés font le bien public, Paris, Vrin, 1974.

Meinecke F. (1936), Die Entstehung des Historismus, München, R. Oldenburg, 1965.

Mercier Ch. (1934), «L'esprit de Calvin et la démocratie», Revue d' Histoire Ecclésiastique, 30 (1), 5 53.

Merton R. K. (1936), «The Unanticipated Consequences of Social Action», repris in R. K. Merton, Sociological Ambivalence and Other Essays, New York, The Free Press, 1976.

Monter W. F. (1976), «The Consistory of Geneva 1559-1569», Bibliothèque d' Humanisme et Renaissance, 38, 467-484.

Popper K. R. (1945), The Open Society and its Enemies, London, Routledge and Kegan Paul.

Smith A. (1759), The Theory of Moral Sentiments, Oxford, Clarendon Press, 1976.

- (1776), An Inquiry into the Nature and Causes of the Wealth of Nations, Oxford, Clarendon Press, 1976.

Weber M. (1904), Die protestantische Ethik und der «Geist» des Kapitalismus. Trad. fr., L'éthique protestante et l' esprit du capitalisme, Paris, Plon, 1964.

- (1906), Kirchen und Sekten. Trad. fr., Les sectes protestantes et l'esprit du capitalisme, Paris, Plon, 1964.

- (1913), Über einige Kategorien der verstehenden Soziologie, repris in Weber (1922 a).

- (1919), Wissenschaft als Beruf. Politik als Beruf, in Weber (1921).

- (1920-21), Gesammelte Aufsätze zur Religionssoziologie, Tübingen, Mohr.

- (1921), Gesammelte politische Schriften, Tübingen, Mohr. Trad. fr. Partielle, Le savant et le politique, Paris, Plon, 1959.

- (1922 a), Gesammelte Aufsätze zur Wissenschaftslehre. Trad. fr. Essais sur la théorie de la science, Paris, Plon, 1965.

- $\quad$ (1922 b), Wirtschaft und Gesellschaft, Tübingen, Mohr, 1972.

- (1923), Wirtschaftsgeschichte. Trad. fr. Histoire économique, Paris, Gallimard, 1991.

- (1924), Gesammelte Aufsätze zur Soziologie und Sozialpolitik, Tübingen, Mohr. 